\title{
Violences de guerre et transmission de la mémoire des conflits à travers l'exemple de la campagne de Calabre de 1806-1807
}

Nicolas Cadet

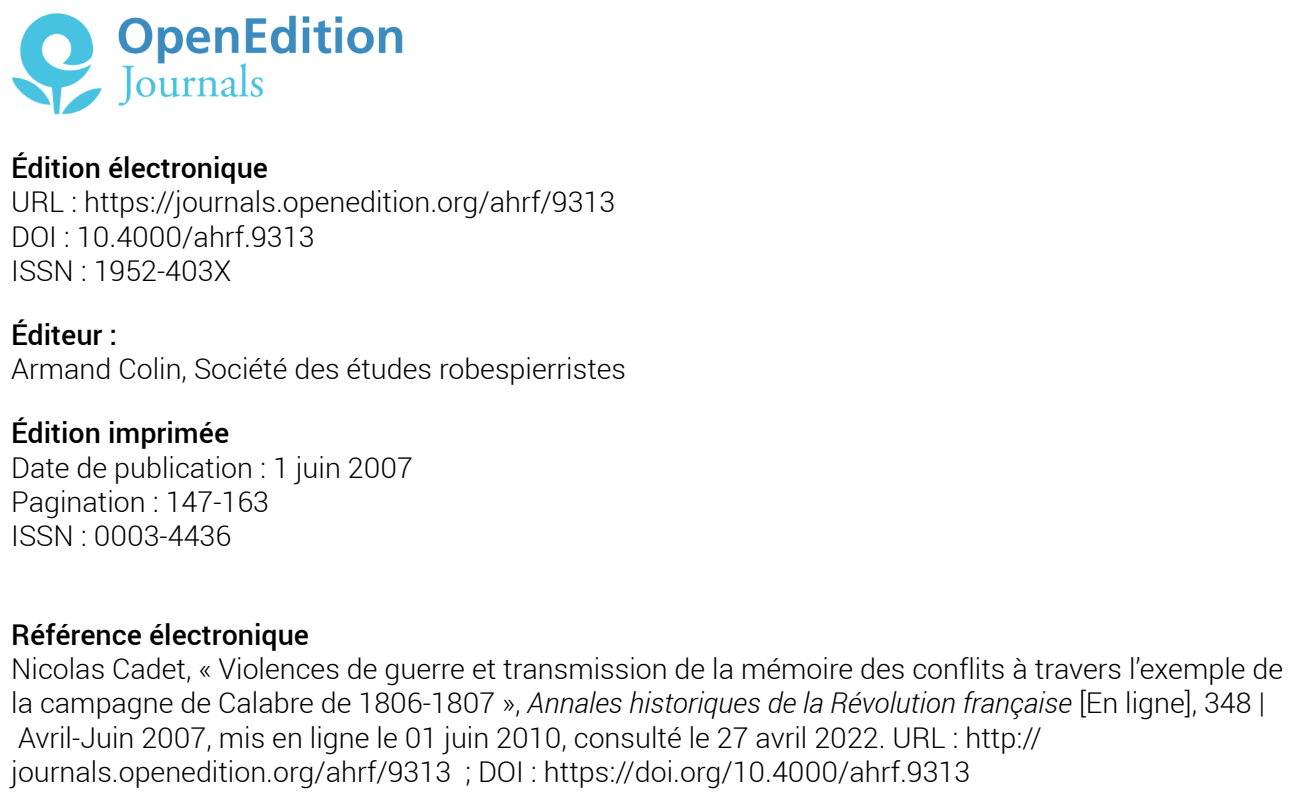

Ce document a été généré automatiquement le 27 avril 2022.

Tous droits réservés 


\title{
Violences de guerre et transmission de la mémoire des conflits à travers l'exemple de la campagne de Calabre de 1806-1807
}

\author{
Nicolas Cadet
}

Depuis une trentaine d'années, en France, de nombreux épisodes du passé donnent lieu à de nouvelles lectures et interprétations qui débouchent sur des débats, des querelles, voire des crises politiques. Ces empoignades prouvent à quel point le lien entre histoire et mémoire reste étroit dans notre pays, et combien ce sujet est propre à exciter les passions. Les événements qui resurgissent inopinément d'un passé parfois fort lointain concernent généralement les périodes agitées ou troubles de notre histoire. Les époques récentes, dont il subsiste de nombreux témoins - l'occupation, le régime de Vichy, la guerre d'Algérie - suscitent des polémiques passionnées qui débouchent souvent sur la mise en place d'une législation spécifique, destinée à offrir une réparation morale aux souffrances subies jadis par telle ou telle partie de la population. Depuis quelques années, cependant, des épisodes de plus en plus éloignés dans le temps font, à leur tour, l'objet d'une relecture attentive et deviennent enjeux de mémoire. Ainsi, après les grands drames des "années noires » et de la décolonisation, c'est maintenant la Première Guerre mondiale, la Révolution, voire le XVIII ${ }^{e}$ ou le XVII ${ }^{e}$ siècle, qui éveillent l'intérêt du public: les mutins de 1917, les victimes des colonnes infernales ou de la colonisation sont placés sous les feux de l'actualité et font l'objet de solennelles réhabilitations. Longtemps considéré par l'opinion comme un véritable sanctuaire de notre gloire nationale, le Premier Empire est à son tour convoqué au tribunal de l'histoire, et sommé de rendre des comptes. Les années 1799-1815 sont, depuis peu, revisitées, réinterprétées, et vivement critiquées. Ainsi, en 2005, le livre de Claude Ribbe, Le crime de Napoléon, établissait une comparaison plus qu'audacieuse et rien moins que contestable entre les massacres perpétrés par l'armée du général Leclerc lors de l'expédition de reconquête de Saint-Domingue (1802), et les génocides mis en œuvre par les nazis entre 1939 et 1945. Précurseurs de Hitler, les Français 
auraient employé les cales de leurs navires comme " chambres à gaz " pour exterminer les populations révoltées des Antilles ${ }^{1}$... Pour contraire aux règles les plus élémentaires de la recherche en histoire que soit la démarche suivie par cet auteur, l'émotion suscitée par cet ouvrage n'en a pas moins eu des conséquences immédiates: les cérémonies prévues pour commémorer le bicentenaire de la bataille d'Austerlitz ont été annulées. Cependant, si le choix de revenir sur l'expédition de Saint-Domingue pour ouvrir le dossier d'accusation du futur empereur découle de la redécouverte du passé colonial de la France et de son rôle dans le commerce des esclaves, il n'est pas fortuit que ce soit à l'occasion d'une des "petites guerres" menées à la période révolutionnaire et impériale que s'ouvre la polémique.

Les années 1792-1815 ont en effet été particulièrement fécondes en conflits insurrectionnels opposant une armée régulière à une population civile en armes: guerre de Vendée (1793-1796), campagne d'Egypte (1798-1801), de Calabre (1806-1807), d'Espagne (1808-1813), du Tyrol (1809), etc. Ce type d'affrontement se caractérise par l'intensité des pratiques de violence mises en œuvre par les deux camps, et se traduit généralement par des massacres en masse de non-combattants Aussi, le souvenir de ces épisodes reste-t-il généralement très présent dans les régions qui en ont été le théâtre, comme le montrent les exemples de la guerre de Vendée ou de l'expédition de SaintDomingue. Certaines des ces «petites guerres» demeurent toutefois dans un oubli profond et leur souvenir semble perdu à tout jamais, à l'instar de la campagne de Calabre de 1806-1807. Initialement conçue comme une simple opération de police, cette expédition menée par la France aux confins de la péninsule italienne débouche sur une violente insurrection populaire soutenue par l'Angleterre, et mobilise durant plus de quinze mois d'importants contingents de l'armée napoléonienne ${ }^{2}$. Au cours de cette campagne, la rudesse des affrontements n'a d'égale que l'ampleur des dommages subis par la population civile. Néanmoins, contrairement à la guerre d'Espagne qu'il préfigure sur de nombreux points, le soulèvement calabrais est aujourd'hui presque totalement oublié dans les pays qui s'y sont trouvés impliqués, malgré le retentissement que ce conflit a pu avoir dans certains d'entre eux sur le moment. Un faisceau complexe de raisons explique l'occultation de cet épisode par notre mémoire collective. Aussi, pour en saisir les mécanismes, convient-il tout d'abord de rappeler brièvement le déroulement de cette guerre et les conditions dans lesquelles elle se déroule, avant d'examiner ses conséquences et son retentissement dans les pays concernés.

3 Le 27 décembre 1805, au lendemain de la signature du traité de Presbourg qui consacre sa victoire sur l'Autriche et la Russie, Napoléon ordonne aux troupes stationnées en Italie du nord de marcher sur le royaume de Naples et de s'en emparer. Officiellement, cette expédition a pour but de châtier les souverains napolitains - le roi Ferdinand IV de Bourbon et sa femme Marie-Caroline-qui, malgré leur promesse de demeurer neutres en cas de guerre opposant la France à ses adversaires européens, ont ouvert leurs ports aux troupes russes et britanniques. En fait, cette opération s'inscrit dans un vaste projet géopolitique : l'empereur entend faire de l'Italie du sud le premier maillon $d u$ « Grand Empire » qu'il rêve de constituer en Europe. Préfigurant la mise en place du système du blocus continental, l'annexion de cette partie de la botte vise également à priver la Grande-Bretagne d'un important partenaire commercial, tout en fournissant aux milieux d'affaires français débouchés et matières premières à bas prix. Enfin, la 
possession de la Sicile fournirait à la France une base capable de rivaliser avec Malte et Gibraltar, et renforcerait la position française en Méditerranée.

4 L'armée chargée d'entreprendre la conquête du royaume de Naples compte plus de 40 000 hommes placés sous le commandement nominal de Joseph Bonaparte, pressenti pour remplacer Ferdinand IV sur le trône. Dans la réalité, la direction des opérations est confiée au maréchal Masséna, vétéran éprouvé des guerres de la Révolution. Dès l'origine, cependant, cette " armée de Naples » souffre de son manque de prestige et de reconnaissance auprès de l'empereur et des autres corps de la Grande Armée. L'Italie méridionale est considérée comme un front périphérique, éloigné, et son occupation est perçue comme une simple opération de police, tant les forces anglo-bourboniennes qui font face aux Français paraissent incapables de s'opposer à leur progression. De fait, l'armée de Ferdinand IV est méprisée par tous les militaires européens, tandis que les régiments britanniques - qualifiés de "débiles bataillons du tyran des mers" par Napoléon - sont tenus en piètre estime par le vainqueur d'Austerlitz. De surcroît, ce dernier affecte à ce théâtre d'opérations les généraux et les officiers qui, pour une raison ou pour une autre, lui ont déplu: Masséna, dont les rapines et l'avidité ont fini par le lasser, le maréchal Gouvion Saint-Cyr, resté trop attaché au régime républicain, le général Reynier, proche ami de Moreau, grand rival de Bonaparte sous le Consulat, etc. Être envoyé à l'armée de Naples constitue donc une sorte de sanction ou de demi disgrâce qui rend illusoire tout espoir de rapide promotion.

Dans un premier temps, les opérations se déroulent conformément aux prévisions de l'empereur. Masséna et Joseph entrent sans coup férir dans Naples le 15 février 1806, contraignant les souverains à fuir le continent pour se réfugier en Sicile, sous la protection de la flotte britannique. Quelques semaines plus tard, le corps de Reynier atteint Reggio, extrémité méridionale de la botte, après avoir dispersé les troupes de Ferdinand IV. Pour les vainqueurs, cependant, les difficultés ne tardent pas à surgir : faute de moyens navals, les Français ne peuvent franchir le détroit de Messine et s'emparer de la Sicile, tandis que dans le nord du royaume, la citadelle de Gaëte, défendue par un général demeuré fidèle à Ferdinand, résiste obstinément. Par ailleurs, les occupants sont bientôt confrontés à l'hostilité grandissante d'une part croissante de la population. Durant le printemps 1806, l'agitation et les troubles sont endémiques en Calabre, sans prendre encore la forme d'un soulèvement général.

6 L'intervention britannique sur le continent précipite toutefois celui-ci. Le 2 juillet 1806 , la flotte anglaise débarque 5000 hommes dans le golfe de Santa-Eufémia, à une centaine de kilomètres au nord de Reggio. Le 4, les troupes de Reynier, rassemblées à la hâte, chargent les régiments du général Stuart disposés sur deux lignes parallèles au littoral. Décimés par le feu meurtrier des fantassins anglais, les Français perdent pied et refluent en désordre. Les conséquences de cette défaite sont catastrophiques: la Calabre toute entière se soulève. Reynier et son adjoint Verdier sont contraints d'évacuer entièrement la province après une retraite menée dans des conditions dramatiques. Encouragée par la cour de Palerme, l'insurrection s'étend vers le nord et menace bientôt la capitale. Début août, cependant, la chute de Gaëte permet à Joseph de dégager des renforts qui, confiés à Masséna, sont immédiatement engagés contre les rebelles. La contre-offensive du maréchal permet en quelques semaines de refouler ces derniers dans le sud de la Calabre. Le soulèvement est cependant loin d'être terminé car les insurgés calabrais ont le soutien des autorités bourboniennes de Sicile et de la flotte britannique. Pour garder le contrôle de la révolte, la cour de Palerme a généralisé le 
système des "masses", sorte de milices populaires encadrées par des officiers bourboniens. La stratégie des «massisti » consiste à épuiser les Français en multipliant débarquements et rassemblements sur tous les points de la Calabre, pour se disperser dès que l'approche des troupes impériales est signalée. Lorsqu'elles sont en position de force, en revanche, les masses lancent de brusques attaques sur les positions tenues par les Français, dans le but, le plus souvent, de couper leurs voies de communication. Leur action est relayée sur le terrain par celle des "comitives", petites bandes fortes de quelques dizaines d'hommes seulement qui pratiquent ce que les Français appelleront en Espagne la "guérilla » : elles harcèlent les détachements en marche, enlèvent les courriers, massacrent traînards et isolés.

7 Pour les troupes de Masséna, la guerre prend rapidement une tournure décousue et épuisante. Aux incessantes marches et contre-marches dans un relief tourmenté contre des rebelles insaisissables, s'ajoutent des combats soudains et meurtriers, mais peu décisifs. Les opérations se déroulent en outre dans un climat d'extrême violence. Celuici découle des très vives tensions socio-économiques que connait la Calabre à l'époque. Cette province, comme le reste du royaume de Naples, vit encore sous le régime féodal instauré au Moyen Âge : la masse des paysans exploite les terres des barons et de la bourgeoisie foncière et ploie sous une cascade de taxes et de droits féodaux. À partir de la seconde moitié du XVIII ${ }^{e}$ siècle, en outre, ce système s'est renforcé au profit de la bourgeoisie rurale ou citadine, au détriment des droits communaux reconnus depuis toujours aux villageois, et cet empiètement s'est effectué le plus souvent de façon brutale. Les paysans calabrais ont donc des conditions d'existence particulièrement rudes, et la Calabre de la fin du XVIII ${ }^{\mathrm{e}}$ siècle vit dans un climat de heurts et de violences perpétuelles. L'insurrection prend ainsi la forme d'une explosion libératoire des rancœurs et des haines accumulées de longue date. Aussi, sous couvert de lutter contre les Français et les "Jacobins", c'est-à-dire les habitants issus des classes aisées ouvertes à l'influence française et favorables à Joseph Bonaparte, les insurgés multiplient meurtres, incendies, pillages et extorsions. Par ailleurs, la société calabraise est profondément imprégnée par un catholicisme marqué par l'influence de la ContreRéforme, qui prend une tournure fortement pénitentielle et doloriste. L'envahisseur est vu comme un impie, un hérétique dont l'intrusion constitue une souillure qui doit être effacée par tous les moyens. Aussi, les Français capturés sont-ils soumis à divers supplices qui visent à mettre en exergue leur nature démoniaque, tels que crucifixions, autodafés, éventrations, mutilations faciales. Les corps ainsi tourmentés sont ostensiblement laissés exposés à la vue de tous. De tels spectacles, qui s'ajoutent à l'épuisement et à la peur, portent l'exaspération des occupants à son comble. Les soldats de Masséna se livrent à des représailles aveugles et féroces : villages incendiés par dizaines, civils massacrés par milliers, viols en masse. Considérés comme des «brigands", et non comme des combattants, les insurgés capturés sont immédiatement exécutés et leurs têtes exposées sur des pieux. À ces conditions fort rudes s'ajoute, pour les combattants de Calabre, le sentiment d'être oubliés et abandonnés par l'empereur. Absorbé par la reprise de la guerre contre les puissances du nord de l'Europe, ce dernier ne s'intéresse guère aux événements d'Italie du sud. En outre, la lutte menée contre les Calabrais s'apparente pour lui à de simples opérations de police contre des bandits, et non à une véritable guerre. Aussi, à partir de l'automne 1806, tandis que leurs camarades de la Grande Armée écrasent l'armée prussienne et moissonnent récompenses et promotions, le sentiment de frustration ressenti par les unités stationnées en Italie du sud va s'accentuant. Dans ces conditions, chacun, du 
simple fusilier jusqu'au maréchal Masséna lui-même, s'efforce pas tous les moyens de quitter cette contrée infernale. Dès le 10 août, Masséna "supplie » Joseph de le rappeler des Calabres ${ }^{3}$, et ne cesse, dès lors, de réitérer sa demande. Parmi les militaires du rang, beaucoup de ceux qui ne parviennent pas à se faire affecter sur un autre théâtre d'opérations basculent dans la folie, sombrent dans la boisson, se suicident. Rien ne témoigne mieux de l'amertume et du dégoût éprouvés par les troupes que ce passage des Mémoires du capitaine Duthilt, officier au 1er léger, dont le régiment a été très malmené au cours de la campagne : «De tant de fatigues, de tant de misères, de tant de pertes éprouvées et de dangers bravés, pas un rayon de gloire, rien que des regrets ${ }^{4}$. En juillet 1807, à l'issue d'un an de guerre, les Français ont, dans l'ensemble, reconquis les positions perdues et mis un terme à l'insurrection. Les troubles et l'insécurité demeurent cependant endémiques durant toute la période napoléonienne. Le bilan de cet affrontement sans véritable vainqueur est effroyable. Sur les 40 ou 45000 soldats envoyés par Napoléon dans le royaume de Naples en 1806, 10000 au moins ont été fauchés par les maladies et le feu des Anglais ou des rebelles. En Calabre, les pertes humaines et matérielles sont considérables. Aux victimes des affrontements, des exactions des insurgés et de la répression s'ajoutent les effets de la surmortalité, liée à la faim, aux maladies, au froid. S'il n'est guère possible de dresser un bilan précis des dommages subis par la population calabraise au cours de l'insurrection, tout du moins peut-on comparer les données dont nous disposons pour l'ensemble de la période 1806-1815. En 1806, si l'on en croit les différents chiffres avancés par les Français, la province compte un peu plus de 800000 habitants. Un recensement effectué en 1813 estime à 779000 personnes la population des deux Calabres ${ }^{5}$. Si nous ignorons sur quelles bases ces évaluations ont été faites, ce qui en affaiblit la portée, du moins ontelles le mérite de fournir un ordre de grandeur qui indique une certaine stagnation de la population calabraise durant la "décennie française ». Naturellement pauvres, les provinces méridionales sortent exsangues du conflit: fermes ravagées, champs laissés en friche, bétail abattu ou dispersé, magasins et entrepôts pillés. Pourtant habitué au rude spectacle des champs de bataille, le général Lamarque est atterré par la vision de populations entières errant sur les chemins ou se terrant dans les forêts, exposées aux rigueurs de l'hiver, après la destruction de leurs villages ${ }^{6}$.

Aussi, le caractère absolu et destructeur de cette guerre pose-t-il immanquablement la question de sa répercussion dans le souvenir des peuples qui y ont pris part. La guerre de Calabre a-t-elle donné lieu en France, en Grande-Bretagne, en Italie à un travail d'évocation et de commémoration? Quel écho cet événement a-t-il trouvé dans la mémoire populaire et dans celle des autorités officielles?

10 En France, évaluer l'onde de choc produite par ce conflit signifie prendre en compte ses effets au plan militaire, puis tenter de mesurer s'il a suscité la curiosité et l'intérêt de l'opinion, pendant et après la période napoléonienne. Au plan militaire, la campagne de Calabre fournit quantité d'enseignements sur les faiblesses de l'armée impériale, sur l'inadéquation de sa tactique face à l'armée anglaise, moderne et dotée d'une grande puissance de feu, et sur les pratiques de la "petite guerre ». Il y a donc matière à amples réflexions de la part de l'empereur et de son état-major. Napoléon, cependant, ne tire aucun enseignement de cette laborieuse campagne, et ne modifie en rien ses conceptions stratégiques ou tactiques dans la perspective des affrontements à venir. Comment expliquer cette absence de retour d'expérience? L'empereur est physiquement très éloigné d'un théâtre d'opérations qu'il ne connaît pas. Du royaume 
de Naples, il ne reçoit que des informations incomplètes et irrégulières. Sa vision de la situation militaire en Italie du sud est en complet décalage avec la réalité, et il tend à sous-estimer systématiquement les difficultés rencontrées sur le terrain. Le souverain est également victime de ses préjugés. Pour lui, les revers s'expliquent par l'incapacité de son frère ou de ses généraux, pas par la pugnacité de l'adversaire ou l'impréparation de la campagne. À aucun moment dans sa correspondance, l'empereur n'exprime l'idée qu'il a commis une grave erreur en envoyant une armée de 40000 hommes guerroyer dans un pays pauvre et mal connu sans lui donner les moyens de mener à bien ses opérations.

11 En outre, le moment se prête mal à une réflexion poussée sur cette campagne. La guerre de Calabre, qui commence au lendemain d'Austerlitz et s'achève lors de la signature du traité de Tilsitt, se déroule alors que l'Empire est à son apogée. Aussi, le formidable écho des triomphes impériaux couvre-t-il la note discordante produite par les événements d'Italie méridionale. Intervient également l'aspect peu spectaculaire des engagements, qui font pâle figure à côté des formidables batailles que Napoléon livre aux Russes et aux Prussiens. Mais surtout, aux yeux des militaires de l'époque, la guerre de Calabre est une guerre "ignoble ", c'est-à-dire dépourvue de noblesse: la seule bataille importante livrée, Santa-Eufémia, est une honteuse défaite, une "déroute» dans le langage du temps, car les troupes ont tourné les talons face à l'ennemi. Les quelques victoires remportées l'ont été contre une armée bourbonienne méprisée dans l'Europe entière. Les rebelles Calabrais, eux, sont des «brigands", pas des soldats, il n'y a donc aucun mérite à les vaincre.

12 Les conséquences de cette absence de réflexion ne se font pas attendre: dès l'année suivante, l'empereur renouvelle son erreur, mais à une toute autre échelle, en envoyant ses troupes en Espagne. Là encore, des corps aux lignes de communications trop étirées, éloignés des autres formations, s'enfoncent à l'extrémité de la péninsule et y sont battus. À de nombreuses reprises également, les assauts français se briseront sur la puissance de feu des fantassins britanniques. Finalement, l'action combinée des forces de Wellington et des « guérillas » viendra à bout de l'armée impériale.

13 Est-ce à dire, pour autant, qu'aucun enseignement n'a été tiré de la campagne de Calabre ? Bien des officiers qui ont combattu dans le royaume de Naples - les généraux Lamarque, Hugo, Reynier, Verdier, Abbé - seront ensuite employés en Espagne et au Portugal. Ils y appliqueront les pratiques de la « petite guerre » expérimentées en Italie du sud : quadrillage du terrain par les colonnes mobiles, emploi de troupes spécialisées dans la contre-guérilla, utilisation de supplétifs indigènes, etc.

Indépendamment de ses répercussions au plan militaire, la guerre de Calabre a-t-elle contribué à faire mieux connaître l'Italie du sud aux Français? Au début du XIX ${ }^{\mathrm{e}}$ siècle, l'extrémité méridionale de la botte est encore très largement une "terra incognita " pour les Européens, fort peu nombreux à s'y être rendus. Dans le domaine scientifique, l'expédition de Calabre est une sorte de campagne d'Égypte à échelle réduite, car l'armée de Reynier compte dans ses rangs l'helléniste Paul-Louis Courier et plusieurs officiers d'artillerie et du génie, armes « savantes ». Ces derniers rédigent quantité de mémoires et rapports sur cette province, dont l'histoire, la géographie physique et humaine, les ressources économiques sont désormais mieux connues. Cependant, à la fin de l'Empire, malgré presque une décennie de présence française dans le royaume de Naples, la Calabre demeure encore très largement ignorée du public. Dans les années qui suivent la chute de Napoléon, paraissent les premiers livres consacrés à la guerre de 
Calabre qui mentionnent les aspects géographiques ou culturels particuliers à cette contrée, notamment celui d'Auguste de Rivarol en 1817, ou du chef de bataillon Duret de Tavel en $1820^{7}$. Ces relations s'ajoutent aux récits de voyages dans le royaume de Naples publiés par Stendhal et Dumas dans les années 1820-1830. Cependant, les ouvrages de ces auteurs fameux reprennent, sans aucun recul critique, les stéréotypes élaborés de longue date sur les Calabrais et réactivés par les Français au cours de la période napoléonienne : les habitants de l'Italie méridionale sont vus comme des gens paresseux, cruels et fourbes, un véritable peuple-enfant incapable de se gouverner luimême. D'autre part, les rares témoignages sur la guerre de 1806-1807 paraissent alors que s'élabore la légende napoléonienne. Ils sont très largement éclipsés par la masse des ouvrages retraçant les campagnes de la Grande Armée, et ne retiennent guère l'attention. Par ailleurs, les récits sur la guerre de Calabre ne cadrent pas avec le goût des lecteurs du XIXe siècle pour l'épopée. Les seuls conflits insurrectionnels qui suscitent l'intérêt des Français sont les guerres de Vendée et d'Espagne, popularisées par des écrivains célèbres tels Balzac ou Hugo. Aussi, jusque dans les dernières décennies du XIX ${ }^{e}$ siècle, l'Italie du sud reste-t-elle encore très largement méconnue du grand public, et personne ne songe à synthétiser ou approfondir les connaissances portant sur cette partie de l'Europe. Il faut attendre les années 1883-1884 pour que soit enfin publiée une somme rigoureuse, dégagée des stéréotypes, qui prenne en compte les apports fournis par les voyageurs européens du XIX siècle, et dresse de l'Italie méridionale un portrait proche de la réalité ${ }^{\text {. }}$

Ce vide laissé par les géographes et les scientifiques sur la Calabre outre un espace dans lequel s'engouffrent les romanciers. C'est en effet au cours de la première moitié du $\mathrm{XIX}^{\mathrm{e}}$ siècle que s'élabore, sous la plume de Stendhal et Dumas, tous deux bons connaisseurs de l'Italie du sud, le mythe romantique du «bandit calabrais ». Stendhal voyage à plusieurs reprises en Italie, notamment en 1827-1828, et publie Rome, Naples et Florence dès $1826^{\circ}$. Dumas manifeste également un grand intérêt pour la partie méridionale de la péninsule, profondément liée à son histoire familiale : de retour de la campagne d'Égypte, son père, le général Dumas, a été emprisonné à Tarente sur ordre de Ferdinand IV. Cette captivité a indirectement entraîné sa mort et la ruine de sa famille. Dumas parcourt la Calabre en 1834-1835 et publie son Voyage en Calabre en $1842^{10}$. Il séjourne de nouveau à Naples à la fin des années 1850 , en vue de se documenter pour son futur roman, La San Felice, paru en 1862, qui a pour cadre la révolution napolitaine de 1799 et la violente réaction royaliste qui l'a suivie. Ces deux auteurs élaborent le mythe du "bandit calabrais", au moment même où Prosper Mérimée édifie celui du «bandit corse » (Matteo Falcone est publié en 1829, Colomba en 1840). Dans les deux cas, le mythe est bâti à partir des mêmes éléments : corse ou calabrais, le «bandit » est un homme fier, libre et indépendant, qui incarne les valeurs d'une société traditionnelle, rurale et patriarcale, imprégnée par la notion d'honneur, bien éloignée du monde matérialiste et emporté par la modernité qui naît au cours de la première moitié $\mathrm{du} \mathrm{XIX}^{\mathrm{e}}$ siècle, et que rejette les romantiques. Dans les années 1830-1840, le bandit calabrais fait figure de héros positif. De fait, on ne trouve pas chez Stendhal ou Dumas les descriptions horrifiées des atrocités commises par les chefs de bandes, comme chez Rivarol ou Duret de Tavel : véritable Robin des bois, le bandit est un dandy justicier qui porte gilet à boutons d'argent et ceinture de soie, un redresseur de torts qui dévalise les riches pour donner aux pauvres.

À partir de la décennie 1860, cependant, l'image du bandit calabrais se brouille et devient plus ambigüe. Les luttes pour l'unité italienne, l'épopée de Garibaldi, attirent 
l'attention du public français sur cette partie de la botte. Quantités d'ouvrages de vulgarisation reprennent l'image du bandit calabrais, notamment ceux de Marc Monnier et d'Émile Normand ${ }^{11}$, mais en insistant davantage sur sa férocité: la description du pittoresque costume et de la panoplie guerrière du brigand demeure incontournable, mais le héros positif, protecteur de la veuve et de l'orphelin, s'efface derrière la brute cruelle et sanguinaire. Dans La San Felice, Dumas consacre un chapitre entier aux "brigands » de 1799, et s'attarde à décrire le terrible Memonne, "l'ogre sanfédiste", qui se délecte à boire du sang dans le crâne de ses ennemis ${ }^{12}$. Dans le même temps, il rédige, mais n'achève pas, un autre monumental roman, Le chevalier de Sainte Hermine, qui a en partie pour cadre la campagne de Calabre de $1806^{13}$. Ces deux ouvrages raniment l'image de férocité et de fourberie qui s'attache à la figure du Calabrais depuis l'occupation française, et s'oppose à celle du bandit-justicier pénétré de loyauté.

Ces mythes, élaborés dans les décennies qui suivent la guerre de 1806, contribuent à ancrer en France l'image d'une Calabre terre de sang et de violence, où tout se règle à coups de fusils et de poignard, topos qui n'a guère évolué depuis. Ce stéréotype se diffuse également en Grande-Bretagne, où le retentissement de la campagne est beaucoup plus important.

En Angleterre, l'onde de choc produite par la campagne de Calabre est facile à mesurer. La nouvelle de la victoire remportée le 4 juillet parvient à Gibraltar vers le 17 août 1806, puis atteint la Grande-Bretagne dans les premiers jours de septembre. Le 3 septembre, le Times titre en capitales "Victory in Calabria » et reproduit la dépêche rédigée par le général Stuart. Le retentissement de la bataille est immense. Première victoire remportée sur le continent européen sur les troupes de Napoléon, Maida - nom donné par les Anglais à cet affrontement - apparaît comme le pendant terrestre de Trafalgar, et renforce le camp des partisans de la poursuite de la guerre contre la France. La nouvelle de ce succès tombe à un moment crucial: depuis plusieurs mois, des négociations sont en cours entre la France et l'Angleterre pour aboutir à un accord de paix. Fox, le premier ministre britannique, est déterminé à mettre fin au conflit qui oppose les deux nations, et il est disposé à faire de nombreuses concessions à Napoléon. Or, les négociations sont rompues en septembre $1806^{14}$. Certes, les causes de cet échec sont plurielles : la mauvaise foi de l'empereur, qui ne souhaite pas réellement la paix et fait traîner en longueur les pourparlers, le refus des Russes de s'accorder avec la France, et, surtout, la mort de Fox, expliquent largement la rupture des discussions entre Français et Anglais. Cependant, il est permis de penser que la nouvelle de la victoire remportée en Calabre contre une armée réputée invincible n'a pu qu'affaiblir davantage le camp des partisans de la paix, et renforcer celui des tenants de la poursuite du conflit.

Du fait de son écho, Maida donne lieu à une série de commémorations officielles et à une abondante production littéraire, poétique, musicale. Honneurs et récompenses pleuvent sur les vainqueurs, notamment sur le général Stuart et ses officiers, qui reçoivent grades et pensions, tandis qu'une médaille est frappée pour commémorer l'événement. Dès 1806, une marche militaire est composée, et jouée à Bath et à Londres $^{15}$. En 1808, un poème monumental qui célèbre le combat dans la veine de l"Iliade est publié par un officier de l'armée des Indes ${ }^{16}$. La production iconographique, pour sa part, se borne principalement à une caricature de James Gillray réalisée le 13 septembre 1806, et à quelques gravures montrant - sous une forme fantaisiste dans la 
plupart des cas - la bataille de Maida. L'éclat de la victoire est rehaussé par le fait que celle-ci a été remportée contre un adversaire plus aguerri, et que les Britanniques ont montré leur esprit chevaleresque en rachetant aux Calabrais leurs prisonniers français afin d'éviter à ceux-ci d'être massacrés. Triomphe des plus honorables, Maida est assimilée aux plus retentissants succès remportés par les Anglais, Azincourt ou Blenheim. Cependant, l'enthousiasme soulevé par cet événement retombe vite. Comme en France, l'émotion suscitée par la petite campagne de Calabre est bientôt éclipsée par la guerre d'Espagne, beaucoup plus longue, meurtrière, et décisive. Néanmoins, l'intérêt pour cette période resurgit en 1848 avec la publication d'un roman en trois volumes, Adventures of an Aide de camp ${ }^{17}$, dont l'action se situe lors de la guerre de Calabre de 1806. Cette fiction sacrifie à tous les impératifs du mode épique et reprend le thème $\mathrm{du}$ bandit calabrais féroce et impitoyable. Toutefois, dans sa préface, l'auteur resitue son roman dans le contexte de la tentative ratée d'unification de l'Italie en 1848-1849, et annonce la libération prochaine de la péninsule du joug autrichien. Enfin, le souvenir de cette page de l'histoire britannique longtemps oubliée semble resurgir depuis une dizaine d'années et s'accompagne de la publication d'assez nombreux ouvrages $^{18}$.

Dans le cas de l'Italie, la transmission de la mémoire de la guerre de Calabre à travers les générations est plus difficile à appréhender. À quelle échelle, en effet, envisager la répercussion de cet événement sur le sol italien ? Celle de la seule Calabre ? Du Mezzogiorno ? De la péninsule tout entière ? Il faut prendre garde à l'anachronisme : à l'époque napoléonienne, l'Italie est une expression géographique. Les différences qui opposent les États de la botte, ceux du nord et du sud en particulier, ne sont pas seulement politiques, mais également géographiques, linguistiques, culturelles. Il est probable que pour un Piémontais ou un Milanais du début du XIX ${ }^{\mathrm{e}}$ siècle, la Calabre est une contrée aussi éloignée et exotique que pour un Français. Le retentissement de l'insurrection de 1806 a dû être pratiquement nul à l'époque, surtout si l'on prend en compte le caractère troublé de la période, et les multiples soubresauts politiques et militaires qu'a connus l'Italie sous l'Empire. Dans ces conditions, il est même permis de se demander si la nouvelle du soulèvement calabrais a été connue en dehors du royaume de Naples.

21 À l'échelle de l'ensemble de l'Italie, la question de l'interprétation de la guerre de Calabre ne se pose qu'à partir du moment où la péninsule tout entière est unifiée par la maison de Savoie dans les années 1860. Les répercussions de l'insurrection calabraise et la façon dont elle est passée dans les mémoires doivent donc être envisagées à l'échelle de l'Italie du sud, et principalement de la Calabre. Il faut également considérer que le soulèvement de 1806-1807 n'est qu'un épisode de la "décennie française » en Italie méridionale, période fort riche en événements et dont les répercussions au plan politique ont été considérables. L'insurrection de 1806, qui succède à celle de 1799 et précède celle de 1820, ne constitue que l'un des rebondissements de ces temps agités, même si l'ampleur qu'elle a revêtue lui confère un relief particulier. Par ailleurs, l'onde de choc de cette guerre doit être envisagée à deux niveaux, car comme tout événement de ce type, le conflit calabrais a donné naissance à une mémoire populaire et à une mémoire officielle, qu'il importe de distinguer.

Pour étudier les traces laissées par cette guerre dans le souvenir, la culture populaire et l'imaginaire des Calabrais, il faut tenter d'évaluer l'intensité de la commotion subie en revenant sur le bilan humain du conflit. Celui-ci semble en effet avoir été suffisamment 
lourd pour que le souvenir de cet événement reste durablement imprimé dans les mémoires. Les conséquences globales des combats sur la démographie calabraise évoquées plus haut ne permettent pas de mesurer avec précision le choc subi par certaines communautés. Or, au cours des assauts lancés contre les localités rebelles, c'est par dizaines, ou par centaines, que les non-combattants sont massacrés : quarante à cinquante morts à Marcellinara en juillet, des centaines à Lauria en août, une quarantaine de personnes-dont des familles entières-à Fiume-Freddo, début septembre... À l'échelle de sociétés numériquement restreintes, ces homicides massifs ont dû être sources de véritables traumatismes, décelables encore des décennies plus tard. La blessure générée dans la population calabraise par ces événements est-elle perceptible dans les années qui suivent ? Comment s'exprime t-elle ? Combien de temps a-t-elle mis à s'atténuer? Pour mesurer en profondeur les formes revêtues par cette commotion, il serait nécessaire de séjourner longuement en Calabre, de recueillir les traditions orales et écrites, de se pencher sur la toponymie, et de comparer le résultat de ces recherches avec les faits. Le regard de l'historien doit, dans ce cas, s'effacer devant celui de l'anthropologue, ou de l'ethnologue, ce qui n'est pas aisé. Je me suis personnellement rendu dans cette région au cours de l'été 2003, mais ce bref séjour, partagé entre les recherches dans les dépôts d'archives et les excursions sur les lieux les plus emblématiques de la guerre de 1806, a été trop limité pour me permettre d'appréhender de manière vraiment convaincante les traces matérielles ou culturelles laissées par cet épisode auprès de la population. Cependant, il m'a semblé que ce conflit était aujourd'hui bien oublié dans la mémoire populaire : nulle fête ou commémoration religieuse n'évoque les combats menés contre les Français, et la présence de l'occupant ne paraît pas avoir laissé de traces dans la toponymie. À l'exception de Dumas, les voyageurs français qui ont parcouru cette contrée au XIX ${ }^{e}$ siècle ne semblent jamais avoir entendu parler de cette guerre. Dans les années 1950, la journaliste milanaise Maria Brandon Albini a sillonné la Calabre, s'intéressant particulièrement aux coutumes et traditions populaires. Elle a minutieusement relevé les contes ou les chansons évoquant le passé de la province sous une forme légendaire ou mythique ${ }^{19}$. Il est significatif qu'aucun d'entre eux ne fasse allusion aux événements de 1806-1807. Cependant, quelques mythes forgés après coup évoquent des épisodes de la guerre de Calabre. Ainsi, les âmes de soldats français brûlés vifs dans l'hôpital de Cosenza en 1806 reviendraient hanter les lieux sous forme de spectres, poussant de lugubres gémissements $^{20}$, la statue de la Vierge Noire de Longobucco aurait miraculeusement protégé la ville de Cassano de l'attaque du chef brigand Santoro ${ }^{21} \ldots$.. Il est ainsi permis de penser que des dizaines de récits similaires ont été élaborés dans les années qui ont suivi l'insurrection, mais que la culture populaire calabraise au XIX ${ }^{e}$ siècle étant avant tout orale, et personne n'ayant pris la peine de les consigner, le souvenir s'en est perdu.

Un autre facteur peut cependant expliquer l'oubli dans lequel semble être tombée la guerre de 1806-1807. Le souvenir de la "seconde guerre de Calabre" est venu se surimposer à celui de l'insurrection de 1806 et a fini par l'éclipser. Pour les Calabrais, la grande guerre du XIX ${ }^{e}$ siècle n'est pas celle menée contre les Français sous Napoléon, mais celle conduite par les " Piémontais ", c'est-à-dire par l'armée de Victor-Emmanuel II, contre les « brigands » bourboniens de Calabre entre 1861 et 1864. Plus récente d'un demi siècle, cette campagne menée par les troupes du nouvel État italien contre les populations méridionales qui résistaient au processus unitaire semble également avoir atteint des seuils de violence très élevés, et s'être durablement imprimée dans les 
mémoires des habitants du Mezzogiorno, au point d'effacer le souvenir des événements de 1806-1807, comme en atteste cet extrait du livre de Maria Brandon Albini :

« Des brigands, on en parle encore ici. Tous, au moins les vieux s'en souviennent, me dit Mario. Ma grand-mère [...] était un des derniers témoins de ces lointains événements de 1860. [...] Grand-Mère racontait que, en ce temps là, l'armée «Piémontaise » pendait les brigands à l'entrée des villages et les y laissait pourrir pendant des semaines à titre d'exemple $»^{22}$.

Des «brigands" de 1806, il n'est en revanche pas fait mention par les Calabrais interrogés par la journaliste. Reste à déterminer si la guerre de Calabre, apparemment oubliée dans la mémoire populaire, a donné lieu à une forme de commémoration officielle. Cette insurrection, qui, après tout, peut être considérée comme un soulèvement «national» contre un envahisseur étranger, à pu être reconnue et célébrée par la monarchie bourbonienne restaurée en 1815, puis, après 1860, par les autorités du nouvel État italien. Ce qui est frappant, cependant, lorsque l'on se trouve en Calabre, c'est de mesurer l'absence totale d'une quelconque prise en compte de ces événements dans l'espace public. À Cosenza, Amantea, Santa-Eufémia, ou encore Soveria-Manelli, lieux emblématiques de l'insurrection, pas le moindre élément de commémoration tel que plaques, stèles, statues, médaillons, ou autres rappelant les combats de 1806. Ces supports matériels évoquant un épisode du passé existent pourtant, mais tous se rapportent au processus d'unification de l'Italie, à l'expédition de Garibaldi dans le Mezzogiorno, en 1860. Parcourant Cosenza, en 1897, l'écrivain anglais George Gissing est frappé par le nombre de statues et de bustes à l'effigie de Cavour, Mazzini ou Garibaldi qui ornent les jardins publics ${ }^{23}$. Ce parti pris commémoratif témoigne de la volonté de la maison de Savoie de faire table rase du passé bourbonien. Il semble évident que l'insurrection de 1806, soulèvement « réactionnaire » en faveur de Ferdinand IV rappelant fâcheusement les luttes récentes contre les derniers partisans de l'Ancien Régime, ne pouvait trouver grâce aux yeux du nouvel État. Peut-on penser que celui-ci a été jusqu'à éradiquer de l'espace public les éventuels monuments élevés entre 1815 et 1860 ? Il ne semble pas que les Bourbons, restaurés en 1815, aient fait de grands efforts pour commémorer le souvenir de l'insurrection de 1806, soit parce que celle-ci s'est finalement achevée par un échec, soit parce que ce régime très conservateur répugnait à célébrer une révolte populaire, quand bien même celle-ci avait éclaté en sa faveur.

En définitive, le souvenir de la guerre de Calabre ne subsiste plus guère que dans les publications érudites publiées à la fin du XIX siècle, ou depuis une trentaine d'années, lorsque des historiens italiens ont redécouvert cette page de leur histoire. À ce titre, l'ouvrage d'Atanasio Mozzillo Cronache della Calabria in guerra, somme de plus de 1350 pages parue en 1972 est peut-être le seul «monument » qui donne tout leur relief aux souffrances endurées par les Calabrais en 1806-180724.

La campagne de Calabre souligne le lien étroit qui existe entre violence de guerre, conception de l'honneur militaire et mémoire. La glorieuse victoire de Maida assure le retentissement de cet épisode en Grande-Bretagne. Son souvenir est aujourd'hui encore préservé dans l'espace public de la capitale britannique: une longue avenue et une station de métro du West End portent le nom de Maida. De l'autre côté du Channel, à l'inverse, le gouvernement impérial se garde bien de donner le moindre écho à ce conflit laborieux et sans noblesse, qui sombre aussitôt dans l'oubli. Bien qu'elle ait constitué un véritable laboratoire des pratiques de lutte anti-insurrectionnelle et qu'elle annonce sur de nombreux points les innombrables guerres coloniales que la 
France livrera au XIX ${ }^{e}$ siècle, la campagne de Calabre n'a pas non plus laissé de traces dans les annales militaires du temps. En Angleterre comme en France, les opérations menées aux confins de l'Italie du sud ne constituent finalement qu'un épisode mineur, un rebondissement sans grande conséquence dans l'affrontement inexpiable qui oppose les deux nations à l'échelle de la planète entière durant un quart de siècle. Plus surprenant est le peu de retentissement qu'a eu cet événement en Italie. À l'inverse de l'Espagne, où le combat mené par le peuple en armes contre l'envahisseur napoléonien est célébré comme l'une des pages les plus glorieuses de l'histoire de la péninsule, l'insurrection calabraise n'est nullement considérée comme une guerre de libération par les Italiens. Cette particularité s'explique moins par l'échec final du soulèvement que par le caractère féroce de la lutte qui oppose les Calabrais entre eux et par la confusion qui en découle. Il est en effet difficile de déterminer qui, des partisans de Ferdinand IV ou des « jacobins » ralliés aux Français, incarnent le droit et la justice. En se soulevant contre l'occupant, les premiers obéissent certes à un réflexe patriotique encore faudrait-il déterminer avec précision ce que recouvre ce terme dans l'Italie du début du XIX ${ }^{e}$ siècle - mais ils défendent un régime archaïque et oppressif représenté par un monarque incapable et peu soucieux du sort de son peuple. Les Calabrais partisans de Joseph, pour leur part, sortent souvent des rangs de cette bourgeoisie foncière qui pressure impitoyablement les paysans, et ils n'hésitent pas à se mettre au service d'un souverain étranger pour préserver leurs intérêts. Une ultime raison, enfin, peut contribuer à expliquer l'oubli dans lequel a sombré la guerre de Calabre : les actes d'une extrême brutalité auxquels se livrent les insurgés - crucifixions, mutilations, lacération des corps, bûchers expiatoires - s'inscrivent dans un langage de la violence qui est de moins en moins admis et compris par leurs contemporains. Ces supplices, qui visent à déshumaniser celui qui les subit en révélant sa nature corrompue et impure, s'intègrent dans l'univers culturel d'une grande partie des Européens, du Moyen Âge jusqu'à la fin de l'époque moderne. Au XVIII ${ }^{e}$ siècle, cependant, les nouvelles formes de sensibilité nées avec le rationalisme des Lumières ne s'accommodent plus de ces pratiques, et leurs auteurs sont considérés avec une réprobation croissante. La Révolution française, les massacres de septembre et les excès de la Terreur cristallisent le dégoût de l'opinion pour ces procédés féroces qui dévoilent le caractère profondément inhumain de ceux qui les commettent. À ce titre, il est significatif que les témoins français de la guerre de Calabre désignent si souvent leurs adversaires sous le terme de "cannibales ». Ainsi, en faisant resurgir d'un passé que l'on espérait révolu ces gestes d'un autre âge, les insurgés ne pouvaient que susciter l'horreur et l'indignation des générations à venir, et, par là, se condamnaient à tomber dans l'oubli.

\section{NOTES}

1.Claude RiBBE, Le crime de Napoléon, éditions Privé, 2005, p 139-143. L'auteur écrit notamment : « Victor Schoelcher lui-même, dans sa Vie de Toussaint Louverture (1889) se déclare informé de ces techniques d'extermination nouvelles qui allaient, hélas, être 
développées au XXe siècle par Hitler pour se débarrasser des populations juives » (p 143).

2.De mars 1806 à juillet 1807, en effet, l'envoi de contingents successifs en Calabre et dans les parties des provinces limitrophes touchées par l'insurrection porte à plus de 20 000 le nombre d'hommes qui ont combattu dans ces régions.

3.AN, 304 Mi45, Lettrre de Masséna à Joseph Castro-Villari, 10 août 1806. L'actuelle région de Calabre est en effet à l'époque divisée en deux provinces séparées par la rivière Netto : la Calabre " citérieure ", au nord, avec Cosenza pour capitale, et la Calabre « ultérieure », au sud, gouvernée depuis Catanzaro.

4.Pierre Charles DuthILT, Mémoires du capitaine Duthilt ou Mes campagnes et mes souvenirs de 1792 à 1815, Tallandier, 1909, p. 250.

5.Celestino RICCI, Picciolo Atlante Geografico-Statistico del Regno di Napoli, Della tipografia di Angelo Trani, 1813, p 47.

6.AN, 566 AP 15. Lettre du général Lamarque à Joseph Bonaparte, Pisciotta, 25 août 1806.

7.Auguste DE RIVAROL, Notice historique sur la Calabre pendant les dernières révolutions de Naples, Paris, Anselin et Pochard, 1817 ; Duret DE TAVEL, Séjour d'un officier français en Calabre ou lettres propres à faire connaître l'état de la Calabre, Paris, Béchet-Ainé, 1820.

8.François LeNORMAND, À travers l'Apulie et la Lucanie, Paris, A. Lévy, 1883, deux volumes. 9.STENDHAL, Rome, Naples et Florence, Paris, Delaunay, 1826.

10.Alexandre Dumas, Voyage en Calabre, éditions Complexe, 1989.

11.Marc MONNIER, Histoire du brigandage dans l'Italie méridionale, Paris, Michel-Lévy frères, 1862 . Émile NORMAND, Les brigands. Épisodes de l'histoire du brigandage dans le royaume de Naples et les États romains, Paris, Bureaux du siècle, 1865.

12.Alexandre DumAS, La San Felice, Gallimard, 1996, p 671-677.

13.Le manuscrit, redécouvert, a été publié en 2005.

14. Thierry LENTZ, Nouvelle histoire du Premier empire, Paris, Fayard, 2002, T 1, ch IX.

15.British Library, H.2818. F (7), «The battle of Maida», marche militaire composée par Ruzzini, paroles de sir George Wallace, imprimée à Dublin en 1806.

16.Lieutenant-colonel SсотT, «The Battle of Maida, an Epic Poem», Londres, 1808.

17.James GRANT, Adventures of an Aide de camp, or a Campaign in Calabria, Londres, $\mathrm{W}$.

Blackwood \& son, 1848.

18.Notamment ceux de Richard Hopton, The Battle of Maida, Fifteen Minutes of Glory, Pen \& Sword books, 2002, et John SteWART, Maida, a Forgotten Victory, Pentland Press, 1997. 19.Maria Brandon ALBINI, Calabre, Arthaud, 1957.

20.Cesare CESARI, L'insurrezione calabrese nel 1806 e l'assedio di Amantea, Commando del corpo di Stato Maggiore, Ufficio Storico, Rome, 1911, p 32.

21.Maria Brandon AlBINI, op. cit., p 97.

22.Maria Brandon ALBINI, op. cit., p 105.

23.George GISsING, Sur les rives de la mer Ionienne. Notes de voyage en Italie du sud, Presses Universitaires du Septentrion, 1997, p 53. L'ouvrage original, By the Ionian Sea est paru chez Chapman and Hall en 1897.

24.Atanasio Mozzillo, Cronache della Calabria in guerra, Napoli, Edizioni scientifiche italiane, 1972, trois volumes, 1351 pages. 


\title{
RÉSUMÉS
}

L'invasion du royaume de Naples par l'armée napoléonienne, en 1806, provoque une vaste insurrection populaire dans les provinces les plus méridionales de la péninsule, notamment en Calabre. Durant près d'un an, les troupes chargées de réprimer le soulèvement s'épuisent à traquer d'insaisissables rebelles qui ne cessent de se dérober à leurs coups, et, avec le soutien des Britanniques, pratiquent une habile stratégie de harcèlement. Pour les soldats français, l'atmosphère de violence extrême dans laquelle se déroule la campagne s'ajoute à son caractère fastidieux et frustrant. Évoluant dans un univers imprégné de religiosité, les Calabrais mènent une véritable croisade contre l'occupant : les sévices et les mutilations qu'ils infligent à ceux qui tombent entre leurs mains entrainent en retour de féroces représailles. Guerre de la violence extrême, la campagne de Calabre souligne le lien étroit qui unit mémoire et modes de représentation. L'insurrection calabraise de 1806-1807 est tombée pour des raisons différentes dans un oubli profond en France, en Italie et en Grande-Bretagne, son souvenir est pratiquement absent de la mémoire officielle comme de la mémoire populaire.

The Violence of War and the transmission of the Memory of Conflicts: the example of the Calabrian Campaign, 1806-1807. The invasion of the Kingdom of Naples by the Napoleonic army in 1806 provoked a major popular insurrection in the southern most provinces of the peninsula, notably in Calabria. For almost one year, the troops entrusted with repressing the uprising exhausted themselves chassing the elusive rebels who always manage to escape, and who with the aid of the British practiced a skillful strategy of harassment. For the French soldiers, the atmosphere of extreme violence in which the compaign developed added to its generally difficult and frustrating nature. Evolving in a universe imbued with religiosity, the Calabrians led a veritable crusade against the occupiers: the violence and multilations they inflicted on whose who fell into their hands elicited in return ferocious reprisals. A war of extreme violence, the Calabrian campaign emphasized the close ties uniting memory and modes of representation. The Calabrian insurrection of 1806-1807 has for different reasons fallen into oblivion in France, Italy, and Great Britain; its memory is practically absent in the official memory as well as in the popular memory.

\section{INDEX}

Mots-clés : mémoire, violences de guerre, honneur

\author{
AUTEUR \\ NICOLAS CADET \\ Tour Atlas, Apt 3010 \\ 10 villa d'Este \\ 75013 Paris \\ nicocadet@wanadoo.fr
}

